Pacific Journal of Mathematics

RINGS OF QUOTIENTS OF RINGS WITHOUT NILPOTEN 


\section{RINGS OF QUOTIENTS OF RINGS WITHOUT NILPOTENT ELEMENTS}

\section{StUART A. Steinberg}

The first theorem gives a number of characterizations of when a ring with zero right singular ideal has a strongly regular right quotient ring. This result (and also Theorem 2 ) is a generalization of a similar theorem of F. W. Anderson for a certain class of lattice-ordered rings and a theorem of G. Renault for reduced rings (i.e., rings without nilpotent elements). As a corollary one obtains a characterization of when a semiprime ring has a strongly regular right quotient ring similar to Utumi's characterization of when a ring has a regular right quotient ring. Also, some theorems on commutative regular rings are extended to strongly regular rings and regular rings that satisfy a polynomial identity. For instance, a reduced ring is regular if and only if each of its prime homomorphic images is regular. This theorem has been obtained independently by Herstein, by Snider, and by Wong. Using rings of quotients some theorems of $R$. Wiegand are also generalized. It is shown that the endomorphism ring $S$ of an ideal $I$ of a strongly regular ring $R$ is strongly regular, and some characterizations of when $R$ is self-injective are obtained.

1. Rings of quotients. We first review some definitions and facts and fix some notation. Good references are [8], [21], [17], and [22]. Let $A$ be a subset of the right $R$-module $M$. Then $r_{R}(A)=$ $r(A)=\{x \in R \mid A x=0\}$ is the right annihilator of $A$ in $R$. Similarly, $l(A)$, where appropriate, will denote the left annihilator of $A$. A ring $R$ is called reduced if it has no nonzero nilpotent elements; i.e., $x \in R$ and $x^{2}=0$ implies $x=0$. If $A$ is a subset of a reduced ring $R$, then $l(A)=r(A)$ will be denoted by $A^{\prime}$. If $A$ is an ideal of a semiprime ring $R$, then, again, $A^{\prime}=l(A)=r(A)$. If $R$ is semiprime, the set of annihilator ideals of $R$ is a Boolean algebra [17, p. 110].

A submodule $N$ of the right $R$-module $M$ is essential in $M$ if $N \cap K \neq 0$ for each nonzero submodule $K$ of $M$. The set of essential submodules of $M_{R}$ is a dual ideal in the lattice of submodules of $M$. The closure of $N$ in $M$ is the submodule $\mathrm{Cl}(N)=\{x \in M \mid x D \subseteq N$ for some essential right ideal $D$ of $R$, and $N$ is closed in $M$ if $N=$ $\mathrm{Cl}(N)$. Since the intersection of a family of closed submodules is closed, the collection of closed submodules of $M$ forms a complete lattice which will be denoted by $C_{r}(M)$. The singular submodule of $M$ is $Z\left(M_{R}\right)=\left\{x \in M \mid r(x)\right.$ is essential in $\left.R_{R}\right\}$ [14]. If $Z\left(M_{R}\right)=0$, then $\mathrm{Cl}(N)$ is closed and is the largest essential extension of $N$ in $M$; and $N$ is closed if and only if $N$ is a complement in $M$-i.e., there is a 
submodule $K$ of $M$ such that $N$ is maximal with respect to $N \cap K=$ $0[8$, p. 61].

A ring $S$ is a right quotient ring of its subring $R$ if for all $x$, $y \in S$ with $y \neq 0$, there exists $r \in R$ with $x r \in R$ and $y r \neq 0$. Each ring $R$ with $l(R)=0$ has a maximal right quotient ring [21]. Throughout this paper $R$ will denote a ring with $l(R)=0$ and maximal right quotient ring $Q=Q(R)$. Let $E$ denote the injective hull of the right $R$-module $R_{R}$. A right ideal $D$ of $R$ is dense if $l_{E}(D)=$ 0 ; equivalently, if $h \in \operatorname{Hom}_{R}(E, E)$ and $h(D)=0$, then $h=0$; equivalently, $Q(D)=Q[8$, p. 58 and §8]. For each $q \in Q$ there is a dense right ideal $D$ of $R$ such that $q D \subseteq R$. If $Z\left(R_{R}\right)=0$, then a right ideal of $R$ is dense if and only if it is essential [21, Theorem 3]; also, in this case, $C_{r}(R)$ contains each right annihilator of $R[8, \mathrm{p} .71$, Proposition 5].

The ring $R$ is regular if for each $a \in R$ there exists $x \in R$ with $a=a x a$; equivalently, each principal left (right) ideal is generated by an idempotent [8, p. 42]. $R$ is strongly regular if it is regular and has no nonzero nilpotent elements; alternatively, each idempotent is central. The ring $R$ has a regular right quotient ring, and then $Q$ is regular, right injective, and the injective hull of $R_{R}$, exactly when $Z\left(R_{R}\right)=0[8, \mathrm{p} .69]$. If $Q$ is regular, then the closure in $Q_{R}$ of each right ideal of $R$ is a summand of $Q_{Q}[8$, p. 70, Theorem 4].

LEMMA 1. Let $R$ be a reduced ring and let $a_{1}, \cdots, a_{n}, x$ be elements of $R$. Let $P$ be a product of $a_{1}, \cdots, a_{n}$ (in any order); let $P_{i}$ be a product of $a_{1}, \cdots, a_{i-1}, a_{i+1}, \cdots, a_{n}$; and let $P_{x}$ be a product of $a_{1}, \cdots$, $a_{n}, x$.

(1) $P=0 \leftrightarrow a_{1} \cdots a_{n}=0$.

(2) If $a_{i}=a_{j}$ for some $i \neq j$, then $P=0 \leftrightarrow P_{i}=0$.

(3) If $P=0$, then $P_{x}=0$.

Proof. Each of these statements is trivial if $R$ is a domain. Since $R$ is a subdirect product of domains [3] each is true when $R$ is merely reduced.

An $f$-ring is a lattice-ordered ring that is a subring and a sublattice of a product of totally ordered rings. The equivalence of (1) and (2) in Theorem 1 has essentially been proven by Anderson [2, Theorem 4.3] for the case in which $R$ is a unital $f$-ring, and the equivalence of (1) through (4) has been proven by Renault [19, Theorem 4.1] for the case in which $R$ is a unital reduced ring.

THEOREM 1. Let $R$ be a ring with zero right singular ideal and maximal right quotient ring $Q$. The following statements are equivalent. 
(1) If $a, b \in R$, then $a R \cap b R=0$ implies $a b=0$.

(2) $Q$ has no nilpotent elements.

(3) Each element of $C_{r}(R)$ is the right annihilator of an ideal of $R$.

(4) Each element of $C_{r}(R)$ is an ideal of $R$.

If $R$ has no nilpotent elements, then (1) is equivalent to each of the following.

(5) If $I$ is a right ideal of $R$, then $I$ is essential in $I^{\prime \prime}$ (and $I^{\prime \prime}$ is the maximal essential extension of $I$ in $R$ ).

(6) If $I$ is a right ideal of $R$, then $I^{\prime}$ is a (unique) right complement of $I$.

Proof. (1) $\rightarrow(2)$ : We first claim that $I+r(I)$ is an essential right ideal of $R$ for each right ideal $I$ of $R$. For if $J$ is a right ideal of $R$ and $[I+r(I)] \cap J=0$, then, since $I \cap J=0$, (1) implies that $I J=0$. Thus $J \subseteq r(I)$, and hence $J=0$; so the claim is proven. Next we show that $R$ is semiprime. Let $I$ be a right ideal such that $I^{2}=0$. Then $I[I+r(I)]=0$, so $Z\left(R_{R}\right)=0$ implies that $I=0$. Next we show that $R$ is reduced. Let $a \in R$ and let $J=r(a R)$. Then $a R J=0$ yields $R J a=0$, and hence $J a=0$, since $R J$ is an ideal of the semiprime ring $R$. If $a^{2}=0$, then $a(a R+J)=0$; so $a=0$ by the essentiality of $a R+J$.

Let $q \in Q$ and let $D$ be a dense right ideal of $R$ for which $q D \subseteq$ $R$. Since $D$ is essential in $R,(q D)^{\prime} \cap D$ is essential in $(q D)^{\prime}$. So $\left[(q D)^{\prime} \cap D\right] \oplus q D$ is essential in $(q D)^{\prime} \oplus q D$, and hence $\left[(q D)^{\prime} \cap D\right] \oplus$ $q D$ is an essential right ideal of $R$. If $x \in(q D)^{\prime} \cap D$, then $x q D=0$; so $x q=0$. Since $q x \in R$ and $(q x)^{2}=0, q x=0$. Thus $q^{2}=0$ implies $q\left(\left[(q D)^{\prime} \cap D\right] \oplus q D\right)=0$, and hence $q=0$. So $Q$ is reduced.

$(2) \rightarrow(1)$ : Let $a, b \in R$ with $a R \cap b R=0$. Let $I$ be the closure of $(a R)_{R}$ in $Q$ and let $J$ be the closure of $(b R)_{R}$ in $Q$. Then $I=e Q$ and $J=f Q$ for some idempotents $e$ and $f$ of $Q$. Since $I$ and $J$ are closed $R$-submodules of $Q_{R}$, and since $a R \cong I$ and $b R \cong J, a \in I$ and $b \in J$. Since $a R$ is essential in $I$ and $b R$ is essential in $J, a R \cap b R=$ 0 implies $I \cap J=0$. Since $I$ and $J$ are ideals of $Q, a b \in I J \subseteq I \cap J=0$.

$(2) \rightarrow(3)$ : If $I \in C_{r}(R)$ and $J$ is a complement of $I$, then $I$ is a complement of $J$. Let $I_{1}$ be the closure of $I$ in $Q$ and let $J_{1}$ be the closure of $J$ in $Q$. Then, as in the previous argument, $I J \subseteq I_{1} J_{1}=0$; i.e., $J \subseteq I^{\prime}$. Since $I \cap I^{\prime}=0$, the maximality of $J$ implies that $J=$ $I^{\prime}$. Similarly, $I=J^{\prime}$, so $I=I^{\prime \prime}$.

That (3) implies (4) is trivial; and that (4) implies (1) follows from the fact that $a b \in \mathrm{Cl}(a R) \mathrm{Cl}(b R) \subseteq \mathrm{Cl}(a R) \cap \mathrm{Cl}(b R)=0$, if $a R \cap$ $b R=0$.

$(1) \rightarrow(5)$ : If $I$ and $J$ are right ideals of $R$ for which $I \cap J=0$, then (1) implies that $J \subseteq I^{\prime}$. Thus $J \subseteq I^{\prime \prime}$ yields $J=0$, and hence $I$ 
is essential in $I^{\prime \prime}$. Since an annihilator is closed, $I^{\prime \prime}$ is the closure of $I$ in $R$.

$(5) \rightarrow(6)$ : If $I \cap J=0$, then, since $I$ is essential in $I^{\prime \prime}, I^{\prime \prime} \cap J=$ 0 . But then $J \subseteq I^{\prime \prime \prime}=I^{\prime}$ since $I^{\prime \prime}$ is an ideal. Since $I \cap I^{\prime}=0, I^{\prime}$ is the unique right complement of $I$.

$(6) \rightarrow(3)$ : Let $I \in C_{r}(R)$. By (6) $I^{\prime \prime}$ is a right complement of $I^{\prime}$ which contains $I$. But $I$ is a right complement of $I^{\prime}$, so $I=I^{\prime \prime}$.

There are reduced rings $R$ for which $Q(R)$ is not reduced. For an example take any domain that is not a right Ore domain.

A right ideal $A$ of the ring $R$ is called an $M$-right ideal provided $x \in A$ whenever there exists a dense right ideal $D$ for which $x D \subseteq A$. In [21, $\S \S 3$ and 4] Utumi has shown that every right complement and every right annihilator in $R$ is an $M$-right ideal, and that $Q$ is regular if and only if each $M$-right ideal of $R$ is a right complement. The following corollary takes this result one step further.

COROLlaRY 1. Let $R$ be a semiprime ring with maximal right quotient ring $Q$. Then $Q$ is strongly regular if and only if each $M$-right ideal of $R$ is an (annihilator) ideal.

Proof. In view of the preceding theorem and Utumi's theorem it suffices to show that each $M$-right ideal $I$ of $R$ is a right complement, assuming that each such $I$ is an (annihilator) ideal. Let $J$ be a right complement of $I$ and let $I_{1}$ be a right complement of $J$ containing $I$. If $I_{1} I^{\prime} \neq 0$, then, since $I$ is essential in $I_{1}, I \cap I_{1} I^{\prime} \neq 0$. This contradicts the assumption that $R$ is semiprime.

Note that this corollary is false without the hypothesis that $R$ is semiprime. For an example take any commutative quasi-Frobenius ring that is not regular.

The next corollary generalizes a well-known fact about integral domains.

CoRollary 2. A ring $R$ has a strongly regular right quotient ring if and only if each of its right ideals has a strongly regular right quotient ring.

Proof. Suppose that $R$ has a strongly regular right quotient ring and let $A$ be a right ideal of $R$. Let $a, b \in A$ with $a A \cap b A=0$. By Theorem 1 , since $Q(R)$ is strongly regular, $a A b A=0$. Thus $a^{2} b^{2}=$ 0 , and hence $a b=0$ by Lemma 1 (2). By Theorem 1, again, $Q(A)$ is strongly regular.

Corollary 2 is false if "strongly" is deleted; for a right ideal 
need not even have a right quotient ring (in the sense of Utumi). To give a concrete example let $R$ be the two-by-two matrix ring over the rationals and let $A=e_{11} R$ be the right ideal of $R$ consisting of those matrices whose second row is zero. Then $A$ is a right injective ring that is clearly not regular [20, Example 5.3].

There is a generalization of Corollary 2, however, which is an immediate consequence of the proof of [8, Theorem 1.2, p. 97]: Let $R$ be a semiprime ring with a regular right quotient ring and let $A$ be a right ideal of $R$ with $l_{A}(A)=0$. Then $A$ is semiprime and has a regular right quotient ring; and

$$
Q(A) \cong e Q(R) e \cong Q\left(\operatorname{Hom}_{R}(A, A)\right),
$$

where $e Q(R)$ is the injective hull of $A_{R}$ in $Q(R)$.

Corollary 3. (Utumi [22, Theorem 1.4].) If a ring $R$ has a strongly regular right quotient ring and a strongly regular left quotient ring, then $Q$ is also the maximal left quotient ring of $R$.

Proof. Since $Q$ is a strongly regular right injective ring it is also left injective (see [21, Theorem 4], for example). Thus it suffices to show that ${ }_{R} R$ is essential in ${ }_{R} Q$. Let $0 \neq q \in Q$. Since $R_{R}$ is essential in $Q_{R}$ there exists $d \in R$ such that $0 \neq q d \in R$. By the leftsided version of Theorem $1 R q d \cap R d \neq 0$, so there exists $a, b \in R$ with $0 \neq a q d=b d$. Let $y \in Q$ with $d y d=d$, and note that $d y=y d$ is idempotent. Then $d a q=d y d a q=d a q d y=d b d y=d y d b=d b \neq 0$, so $R q \cap R \neq 0$.

Next we impose chain conditions on the reduced ring $R$ to obtain a ring theoretic generalization of a theorem about $f$-rings which is due to Anderson [1, Theorems 6.1 and 6.2] (also see [19, Proposition 5.3]).

Lemma 2. Let $A$ be an ideal of the reduced ring $R$. The following statements are equivalent.

(1) $A^{\prime}$ is a maximal annihilator of $R$.

(2) $R / A^{\prime}$ is a domain.

(3) $A$ is a domain.

Proof. If $A$ is not a domain there are nonzero elements $a, b \in A$ such that $a b=0$. If $J$ is the ideal of $R$ generated by $A^{\prime} \cup\{a\}$, then $A^{\prime} \sqsubseteq J^{\prime \prime} \varsubsetneqq R$, since $a \in J^{\prime \prime} \backslash A^{\prime}$ and $b \notin J^{\prime \prime} \quad\left(b \in A^{\prime \prime} \cap a^{\prime}=J^{\prime}\right)$; hence (1) implies (3). That (3) implies (2) is straightforward (and known). If $R / A^{\prime}$ is a domain, then $a \notin A^{\prime}$ implies $a^{\prime} \cap A^{\prime \prime} \subseteq A^{\prime} \cap A^{\prime \prime}=0$. Therefore, $\left(\{a\} \cup A^{\prime}\right)^{\prime \prime}=\left(a^{\prime} \cap A^{\prime \prime}\right)^{\prime}=R$; so $A^{\prime}$ is a maximal annihilator and (2) implies (1). 
THEOREM 2. Let $R$ be a reduced ring with the maximum condition on annihilators. Then $Q=Q(R)$ is reduced if and only if $R$ is a right Ore ring. If this is the case $Q$ is the classical right quotient ring of $R$ and it is a direct sum of division rings.

Proof. Since the Boolean algebra $\mathscr{A}$ of annihilators of $R$ has the maximum condition, it is finite. Let $A_{1}, \cdots, A_{n}$ be the maximal elements of $\mathscr{A}$. Then $\bigcap A_{i}=0$. If $B_{i}=A_{i}^{\prime}$, then $B_{i} \cap \sum_{j \neq i} B_{j} \subseteq$ $B_{i} \wedge\left(\vee_{j \neq i} B_{j}\right)=\vee_{j \neq i} B_{i} \wedge B_{j}=0$, where the lattice operations are taken in $\mathscr{A}$. Thus $B=\sum \oplus B_{i} \subseteq R$. Since $B^{\prime}=0 B$ is essential in $R$. Since each $B_{i}$ is a domain $B$ has regular elements, and since $B^{\prime}=0$ the regular elements of $B$ are still regular in $R$.

If $R$ is right Ore, then so is $B$, and $R$ and $B$ have the same classical right quotient ring $T$. Since $T$ is a direct sum of division rings it is equal to $Q$.

Suppose, conversely, that $Q$ is reduced. Let $a$ and $x$ be elements of $R$ with $x$ regular. If $a B_{i}=0$ let $b_{i}$ be any nonzero element of $B_{i}$. If $a B_{i} \neq 0$, then the regularity of $x$ implies that $a B_{i} x R \neq 0$. By Theorem 1, then, $a B_{i} \cap x R \neq 0$. Let $b_{i} \in B_{i}$ with $0 \neq a b_{i} \in x R$. Then $b=b_{1}+\cdots+b_{n}$ is a regular element of $R$ and $a b \in x R$. Thus $R$ is a right Ore ring.

In [20, Theorem 3.5] we have shown that if $R$ is an $f$-ring in which each right ideal that has a finite number of positive generators has a single positive generator, then $Q$ is an $f$-ring extension of $R$. This has motivated the next result.

THEOREM 3. If $R$ is a reduced ring in which all finitely generated right ideals are principal, then $Q$ is strongly regular.

Proof. It is well-known that a domain $S$ can be embedded in a unital domain. (Let $S_{1}$ be the ring obtained from $S$ by freely adjoining the integers to $S$. Then $S_{1}$ is semiprime and $S_{1} / S^{\prime}$ is a domain.) Since $R$ is a subdirect product of domains it can be embedded in a unital reduced ring $T$. Let $R_{1}$ be the subring of $T$ generated by $R$ and 1.

We now show that $Q$ is reduced. Suppose that $q \in Q$ with $q^{2}=$ 0 , and let $d \in R$ with $q d \in R$. Then $q d R_{1}+d R_{1}=c R_{1}$ for some $c \in R$. Hence there exist elements $x_{1}, y_{1}, a_{1}$, and $b_{1}$ in $R_{1}$ such that

$$
\begin{aligned}
(q d) x_{1}+d y_{1} & =c \\
d & =c a_{1} \\
q d & =c b_{1} .
\end{aligned}
$$




$$
c b_{1} y_{1} b_{1}=q d y_{1} b_{1}=q\left[\left(c-q d x_{1}\right) b_{1}\right]=q c b_{1}=q^{2} d=0 .
$$

Since $R_{1}$ is reduced, Lemma 1 (2) implies that $c b_{1} y_{1}=0$. Thus $q d y_{1}=0$ and hence $q c=0$. Consequently $q d=0$, and, since $D=\{d \in R \mid q d \in R\}$ is a dense right ideal of $R$, necessarily $q=0$.

Corollary 4. (Amitsur [1, p. 62], Cohn [7, Theorems 3.3, 4.1, and 4.2].) An integral domain in which each finitely generated right ideal is principal is a right Ore domain.

The following corollary is an immediate consequence of Theorem 3.

COROLlary 5. If $R$ is a reduced ring in which each finitely generated one-sided ideal is principal, then $Q$ is also the maximal left quotient ring of $R$.

If $Q$ can be made into an $f$-ring extension of the $f$-ring $R$, then $R$ is called a $q f$-ring.

COROLlary 6. A semiprime f-ring $R$ in which each finitely generated right ideal is principal is a qf-ring.

Proof. Since $R$ is reduced [4, p. 63, Theorem 16], $Q$ is strongly regular. Thus Theorem 1 and the nonunital version of [2, Theorem 4.3] imply that $R$ is a $q f$-ring.

We close this section with a short proof of the following result which has been proven by J. Georgoudis for the case in which $R$ is an $f$-ring [11, Proposition 7]. (It is valid even if $S$ is not a faithful extension of $R$.) The largest subring of $Q$ that is a left quotient ring of $R$ is the maximal two-sided quotient ring of $R$ [25, Theorem 8].

Proposition 1. A two-sided quotient ring $S$ (in particular, the maximal two-sided quotient ring) of a reduced ring $R$ is reduced.

Proof. Let $s \in S$ with $s^{2}=0$, and let $D_{1}\left(D_{2}\right)$ be a dense left (right) ideal of $R$ with $D_{1} s \subseteq R\left(s D_{2} \subseteq R\right)$. If $d_{1} \in D_{1}$ and $d_{2} \in D_{2}$, then $d_{1} s^{2} d_{2}=$ 0 and Lemma 1 (3) imply that $\left(d_{1} s d_{2}\right)^{2}=0$. Thus $D_{1} s D_{2}=0$, so $s=0$.

2. Strongly regular rings. The next theorem, which has also been obtained by Herstein, by Snider [10], and by Wong [24], characterizes regular rings among the class of reduced rings. Its motivation is the commutative version given in [16, p. 61]. Our proof is of interest because it is quite similar to that of the commutative version given in [16]. R. Snider has given a counterexample to show that the theorem is not true for regular rings (see [10]), and the theorem 
has been obtained by Fisher and Snider [10] as a corollary of a characterization of regular rings.

If $P$ is a prime ideal of a ring $R$, then the intersection of a maximal chain of prime ideals contained in $P$ is a minimal prime ideal. Recall that a minimal prime ideal of a reduced ring is completely prime; i.e., the quotient is a domain [3]. (A prime ideal in a reduced ring need not be completely prime. For example, let $S$ be a right primitive domain that is not a division ring. Then there exists a division ring $D$ and a subring $R$ of $S$ such that the two-by-two matrix ring over $D$ is a homomorphic image of $R$ [11, Theorem 2.14, p. 43].)

Lemma 3. Let $P$ be a prime ideal of the reduced ring $R$. Then $P$ is a minimal prime if and only if $x^{\prime} \nsubseteq P$ whenever $x \in P$.

Proof. Suppose the condition is satisfied and let $N$ be a minimal prime ideal contained in $P$. If $x \in P \backslash N$ there exists $y \notin P$ such that $x y=0$. This contradicts the fact that $N$ is completely prime, so $N=P$.

Suppose conversely that $P$ is a minimal prime and let $x \in P$. Let $M=R \backslash P$ and let

$$
S=M \cup\left\{a_{1} x a_{2} x \cdots a_{n} x a_{n+1} \mid a_{i} \in M \text { and } n \geqq 1\right\} .
$$

Then $M$ is a multiplicatively closed subset of $R$ maximal with respect to excluding 0 , and $S$ is a multiplicative subset properly containing $M$. Thus $0=a_{1} x a_{2} \cdots a_{n} x a_{n+1}$ for some $a_{i} \in M$. By Lemma 1(1) $a_{1} a_{2} \cdots a_{n} x=0$, so $x^{\prime} \nsubseteq P$.

THeOREM 4. A reduced ring is regular if and only if $R / P$ is regular for each prime ideal $P$ of $R$.

Proof. Suppose that for each prime ideal $P$ of $R R / P$ is regular. Since a regular domain is a division ring, each minimal prime is a maximal left ideal, and therefore each prime is minimal. Let $0 \neq$ $a \in R$ and let $J=a^{\prime}$. Then $\bar{\alpha}$ is a regular element in the reduced ring $\bar{R}=R / J$. Suppose that $\bar{R}$ has an identity element. If $\overline{R a} \varsubsetneqq \bar{R}$ let $\bar{A}$ be a maximal left ideal containing $\overline{R \alpha}$, and let $\bar{P}$ be a primitive ideal contained in $\bar{A}$. Then $\bar{P}=\bar{A}$ and hence $\bar{a} \in \bar{P}$. This contradicts Lemma 3 which implies that $\bar{P}$ consists of zero divisors.

If $\bar{R}$ does not have an identity, then, by a change of notation, we have $a$ is a regular element of $R$ and $1 \notin R$. Let

$$
S=\left\{a^{n_{1}}\left(1-x_{1}\right) a^{n_{2}}\left(1-x_{2}\right) \cdots a^{n_{k}}\left(1-x_{k}\right) \mid k \geqq 1, n_{i} \geqq 1 \text { and } x_{i} \in R\right\} \text {. }
$$

Then $S$ is a multiplicatively closed subset of $R$. If $0 \in S$, then, since 
$R$ is embeddable in a unital reduced ring, Lemma 1 (2) implies that $a(1-x)=0$ for some $x \in R$; hence $x$ is the identity of $R$. If $0 \notin S$, and $P$ is an ideal maximal with respect to being disjoint from $S$, then $P$ is prime, so $R / P$ is a division ring. If $e+P$ is the identity of $R / P$, then $a(1-e) \in P$ gives the contradiction.

We finish up by using rings of quotients to generalize some theorems of $R$. Wiegand [23] on commutative regular rings to strongly regular rings. If $B(S)$ is the set of central idempotents of the ring $S$, then $B(S)$ is a generalized Boolean algebra with lattice operations $e \wedge f=e f$ and $e \vee f=e+f-e f$. (It is a Boolean algebra if $1 \in S$.) The following lemma, though undoubtedly known, is included for completeness.

LEMma 4. Let $R$ be a semiprime subring of $S$, and suppose that $S_{R}$ is an essential extension of $R_{R}$. If $A$ is an ideal of $R$, then $B$, the closure of $A_{R}$ in $S_{R}$, is an ideal of $S$.

Proof. Let $b \in B$ and $s \in S$. Since $R_{R}$ is essential in $S_{R}$, there exists an essential right ideal $D$ of $R$ such that $b D \cong A, s D \subseteq D$, and $s b D \subseteq R$. So $b s D \subseteq b D \subseteq A$; hence $B$ is a right ideal of $S$. Also $s b D A^{\prime}=0$, so $s b D \cong A^{\prime \prime}$. Since $A_{R}$ is essential in $A^{\prime \prime}, D_{1}=$ $\{d \in D \mid s b d \in A\}$ is an essential right ideal of $R$. Thus $s b \in B$ and $B$ is a left ideal of $S$.

The next result appears in [9, p. 90, Theorem 11.9] for the case that $R$ is a commutative semisimple ring with unity.

PRoposition 2. Let $R$ be a regular ring with maximal right quotient ring $Q$. Then $B(Q)$ is the completion of $B(R)$ if and only if each nonzero annihilator ideal of $R$ contains a nonzero central element.

Proof. Since the center of $R$ is contained in the center of $Q$ [21, 1.3], $B(R) \subseteq B(Q)$. Since $Q$ is right injective each annihilator ideal $A$ of $Q$, being a closed right ideal, is a summand of $Q_{Q}$, and hence is a ring summand of $Q$. Thus $B(Q)$ is isomorphic to the Boolean algebra of annihilator ideals of $Q$, and, in particular, $B(Q)$ is complete. In [5] it is shown that the maximal quotient ring of a Boolean ring $B$ coincides with the completion of $B$ considered as a generalized Boolean algebra. So $B(Q)$ is the completion of $B(R)$ if and only if it is a quotient ring of $B(R)$.

Suppose that $B(Q)$ is the completion of $B(R)$. Let $A$ be a nonzero annihilator ideal of $R$ and let $B$ be the closure of $A$ in $Q_{R}$. By Lemma $4 B$ is an ideal of $Q$, so $B=e Q$ for some $e \in B(Q)$. If $f \in B(R)$ with 
$0 \neq e f \in B(R)$, then $e f \in e Q \cap R=A$ [8, p. 70, Theorem 4].

Assume, on the other hand, the condition of the proposition. Let $0 \neq e \in B(Q)$ and let $A=R \cap e Q$. Then $A=[R \cap(1-e) Q]^{\prime}$, so $A$ contains a nonzero central element $a$. Since $R$ is regular there exists $b \in R$ with $a=a b a$. Then $a b=b a=f \in B(R)$ and $a R=f R$. So $0 \neq$ $f=e f$ and hence $B(Q)$ is a quotient ring of $B(R)$.

A ring $R$ is said to satisfy a polynomial identity if there exists a (homogeneous multilinear) polynomial $f\left(x_{1}, x_{2}, \cdots, x_{n}\right)=\sum_{i} w_{i} x_{i_{1}} x_{i_{2}} \cdots x_{i_{n}}$ in noncommuting indeterminates $\left\{x_{j}\right\}$ (where $i$ ranges over the symmetric group $S_{n}$ and $w_{i}$ lies in the centroid of $R$ ) such that $f\left(r_{1}, r_{2}, \cdots, r_{n}\right)=0$ for all $r_{1}, r_{2}, \cdots, r_{n} \in R$ and the kernel of $w_{1}=0$. Such a ring is called a P.I. ring.

COROLlaRY 7. If $R$ is strongly regular or a regular P.I. ring, then $B(Q)$ is the completion of $B(R)$.

Proof. If $R$ is strongly regular this is obvious. In the other case, the conclusion is a consequence of the following theorem of L. Rowen [18, Theorem A]: Each nonzero ideal of a semiprime P.I. ring contains a nonzero central element.

If $R$ is a regular ring, then $B(Q)$ need not be the completion of $B(R)$. For instance, if $R$ is the ring of linear transformations of finite rank of an infinite dimensional vector space (or any regular prime ring without an identity element), then $B(R)=0$ while $B(Q)=$ $\{0,1\}$. On the other hand, it is clear from the proof of Proposition 2 that if $R$ is any semiprime ring with zero right singular ideal in which each nonzero annihilator ideal contains a nonzero central idempotent, then $B(Q)$ is the completion of $B(R)$. Included in this class of rings are the biregular rings and the semisimple weakly reducible rings (see [21, p. 13]). (A ring $R$ is biregular if each principal ideal is generated by a central idempotent. An ideal of a ring is called a matrix ideal if it is a matrix ring of finite degree over a unital reduced ring in which each nonzero one-sided ideal contains a nonzero central idempotent. A ring is semisimple weakly reducible if every nonzero ideal contains a nonzero matrix ideal.)

Proposition 3. The following statements are equivalent for a regular ring $R$ for which $B(Q)$ is the completion of $B(R)$.

(1) $B(R)$ is complete.

(2) Each ideal of $R$ is an essential submodule of a principal right (left) ideal.

(3) $R$ is unital and each annihilator ideal is a summand. 
Proof. To see that (1) implies (2) let $I$ be an ideal of $R$ and let $e Q$ be the closure of $I$ in $Q_{R}$. By Lemma $4 e \in B(Q)=B(R)$, so $I$ is essential in $e R$. Trivially (2) implies that $R$ is unital and, since an annihilator ideal is closed, it is a summand. Finally, (3) implies that $B(R)$ is isomorphic to the Boolean algebra of annihilator ideals of $R$, and hence it is complete.

For the sequel, let $I$ be an ideal of the regular ring $R, \hat{I}=e Q$ the closure of $I$ in $Q_{R}, S=\operatorname{Hom}_{R}\left(I_{R}, I_{R}\right)$ and $T=\operatorname{Hom}_{R}\left(\hat{I}_{R}, \hat{I}_{R}\right)$. Then, since $Z\left(I_{R}\right)=0, S$ is embedded naturally in $T$; and $T \cong e Q e$, $S \cong S_{1}=\{e q e \mid e q e I \cong I\}[8$, p. 97, Theorem 1.2].

Theorem 5. Let $I$ be an ideal of the strongly regular ring $R$. Then $S=\operatorname{Hom}_{R}\left(I_{R}, I_{R}\right) \cong \operatorname{Hom}_{R}\left({ }_{R} I,{ }_{R} I\right)$ is strongly regular.

Proof. Let $a \in Q$ and let $b \in Q$ with $a b a=a$. Let $a_{*}=a b=b a$ and let $\widetilde{a}=b a b$. Then $a_{*}$ is the unique idempotent of $Q$ with $Q a=$ $Q a_{*}$, and $\widetilde{a}$ is the inverse of $a$ in $Q a$. If $c \in Q$, then, since each onesided ideal of $Q$ is an ideal,

$$
Q a c=Q a Q c=Q a_{*} Q c_{*}=Q a_{*} c_{*} .
$$

Thus ${ }_{*}(\sim)$ is a multiplicative (anti-multiplicative) mapping of $Q$ which leaves each idempotent fixed. If $a \in I$, then, since $R$ is regular, $a_{*}$, $\widetilde{a} \in I$. We claim that for $a \in Q, a I \leqq I$ if and only if $a_{*} I \leqq I$. For if $c \in I$, then $a c \in I$ if and only if $a_{*} c_{*}=(a c)_{*} \in I$; if and only if $a_{*} c_{*} R \subseteq I$; if and only if $a_{*} c \in I$. Note, also, that since $Q \alpha=Q \widetilde{a}$, $a I \subseteq I$ if and only if $\widetilde{a} I \subseteq I$.

We will now show that $S$ is regular. Take $a e \in S_{1}$ and let $g=$ $a_{*} e$. Then $g \in S_{1}$ since $a e I \subseteq I$. We claim that $S_{1} g=S_{1} a e$. Since $a e=a e a_{*} e=a e g, \quad S_{1} a e \subseteq S_{1} g$. However, $g=a_{*} e=\tilde{a} e a e \in S_{1} a e$ since $\widetilde{a} e \in S_{1}$; so $S_{1} g \subseteq S_{1} a e$. Thus $S_{1}$, and hence $S$, is regular.

Since $a I \subseteq I$ if and only if $I a \subseteq I, S \cong \operatorname{Hom}_{R}\left({ }_{R} I,{ }_{R} I\right)$.

With the same notation as above let $\mu_{I}: R \rightarrow S$ be the homomorphism given by $\mu_{I}(x)$ is left multiplication by $x$. Note that $S$ is a right $R$-module: If $s \in S, r \in R$, and $a \in I$, then $(s r) a=s(r a)$.

THEOREM 6. Let $I$ be an ideal of the unital regular ring $R$. Let $S=\operatorname{Hom}_{R}\left(I_{R}, I_{R}\right)$ and let $\mu_{I}: R \rightarrow S$ be the left multiplication map. Then $S_{R}$ is projective if and only if $I^{\prime}$ is a summand of $R$ and $\mu_{I}$ is onto.

Proof. Let $\mu=\mu_{I}$ and let $L_{e}$ denote left multiplication by $e\left(L_{e}=\mu(1)\right)$. The actions of $R$ on $S$ and of $L_{e} \cdot R$ on $S$ correspond 
in the natural way. In particular, the $R$-submodules and the $L_{e} \cdot R$ submodules of $S$ are the same. By the proof of [8, Theorem 1.2, p. 97] $T$ is the maximal right quotient ring of $\mu(I)$. Since $\mu(I) \subseteq$ $L_{e} \cdot R \subseteq S \subseteq T, L_{e} \cdot R$ is an essential submodule of $S_{R}$.

Consider the right $R$-module sequence

$$
0 \longrightarrow I^{\prime} \longrightarrow R \longrightarrow L_{e} \cdot R \longrightarrow 0 \text {. }
$$

If $S_{R}$ is projective, then by [15, Lemma 4] $L_{e} \cdot R$ is a summand of $S$. Thus $L_{e} \cdot R=S$ and $(x)$ splits; i.e., $I^{\prime}$ is a summand of $R$. Conversely, if $I^{\prime}$ is a summand and $L_{e} \cdot R=S$, then $(x)$ splits and hence $S_{R}$ is projective.

COROLlaRY 8. Let $R$ be either a unital strongly regular ring or a unital regular P.I. ring. Then the following conditions are equivalent.

(1) $R$ is self-injective.

(2) $\mu_{I}: R \rightarrow \operatorname{Hom}_{R}\left(I_{R}, I_{R}\right)$ is epic for each ideal $I$ of $R$.

(3) $B(R)$ is complete and $I^{\prime \prime} \cong \operatorname{Hom}_{R}\left(I_{R}, I_{R}\right)$ for each ideal $I$ of $R$.

(4) $\operatorname{Hom}_{R}\left(I_{R}, I_{R}\right)$ is a projective $R$-module for each ideal $I$ of $R$.

Proof. We first give a proof assuming that $R$ is strongly regular. (1) $\leftrightarrow(2)$ : If $I$ is an ideal of $R$ and $\phi \in \operatorname{Hom}_{R}\left(I_{R}, R_{R}\right)$, then, since $R$ is regular, $\phi(I) \subseteq I$. But $R$ is injective if and only if every such $\phi$ is the restriction of a left multiplication map of $R$ [6, Theorem I.3.2].

$(1) \rightarrow(3)$ : By Corollary $7 B(R)$ is complete. Since $R=I^{\prime} \oplus I^{\prime \prime}$,

$$
I^{\prime \prime} \cong \mu_{I}\left(I^{\prime \prime}\right)=\mu_{I}(R)=\operatorname{Hom}_{R}\left(I_{R}, I_{R}\right) \text {. }
$$

$(3) \rightarrow(4)$ : By Proposition $3 I^{\prime}$ is a summand of $R$. Since $I^{\prime \prime} \cong$ $\operatorname{Hom}_{R}\left(I_{R}, I_{R}\right), \mu_{I}$ is epic. By Theorem $6 \operatorname{Hom}_{R}\left(I_{R}, I_{R}\right)$ is a projective $R$-module.

(4) $\rightarrow(2)$ : This implication follows from Theorem 7 .

Now if $R$ is a regular P.I. ring, then the only part of the preceding proof that needs modification is the implication $(2) \rightarrow(1)$. Let $J$ be a right ideal of $R$ and let $\phi \in \operatorname{Hom}_{R}(J, R)$. If $K$ is a complement of $J$, then $J \oplus K$ is an essential right ideal of $R$ and $\phi$ can be extended to $J \oplus K$. Thus we may assume that $J$ is an essential right ideal of $R$. By [18, Theorem 6], $J$ contains an essential ideal $I$ of $R$. Then the restriction of $\phi$ to $I$ is given by left multiplication by some element $x$ of $R$. If $a \in J$, then, since $I_{R}$ is essential in $J$, there is an essential right ideal $D$ of $R$ such that $a D \cong I$. So $\phi(\alpha) d=$ $\phi(a d)=x a d$ for each $d \in D$. Hence $\phi(a)=x a$, and so $R$ is right injective. But then $R$ is also left injective since $Q(T)$ is the maximal left quotient ring of any semiprime P.I. ring $T$ [18, Theorem 5]. 
Added in proof. The following example shows that the analogue of Theorem 5 is false for regular P.I. rings; i.e., the endomorphism ring of an ideal of a regular P.I. ring need not be regular (but by Martindale's theorem [18] it is P.I., since it is a subring of $Q$ ). Let $R$ be the ring of all sequences of $2 \times 2$ matrices over the field $F$ which are eventually in $\left(\begin{array}{cc}F & 0 \\ 0 & F\end{array}\right)$, and let $I$ be the ideal of all sequences that are eventually in $\left(\begin{array}{ll}F & 0 \\ 0 & 0\end{array}\right)$. Then $Q$ is the ring of all sequences of $2 \times 2$ matrices over $F[21,2.1]$, and $S \cong\{q \in Q \mid q I \cong I\}=\{q \in Q \mid q$ is eventually in $\left.\left(\begin{array}{cc}F & F \\ 0 & F\end{array}\right)\right\}$.

\section{REFERENCES}

1. S. A. Amitsur, Remarks on principal ideal rings, Osaka Math. J., 15 (1963), 59-69. 2. F. W. Anderson, Lattice-ordered rings of quotients, Canad. J. Math., 17 (1965), 434-448.

3. V. Andrunakievic and Ju. M. Rjabuhin, Rings without nilpotent elements, and completely simple ideals, Soviet Math. Dokl., 9 (1968), 565-567.

4. G. Birkhoff and R. S. Pierce, Lattice-ordered rings, An. Acad. Brasil. Ci., 28 (1956), 41-46.

5. B. Brainerd and J. Lambek, On the ring of quotients of a Boolean ring, Canad. Math. Bull., 2 (1959), 25-29.

6. H. Cartan and S. Eilenberg, Homological Algebra, Princeton University Press, Princeton, 1956.

7. P. M. Cohn, Noncommutative unique factorization domains, Trans. Amer. Math. Soc., 109 (1963), 313-331.

8. C. Faith, Lectures on Injective Modules and Quotient Rings, Springer Verlag, New York, 1967.

9. N. J. Fine, L. Gillman, and J. Lambek, Rings of Quotients of Rings of Continuous Functions, McGill University Press, Montreal, 1965.

10. J. Fisher and $R$. Snider, On the von Neumann regularity of rings with regular prime factor rings, to appear, Pacific J. Math.

11. J. Georgoudis, Two-sided rings of quotients of f-rings, to appear.

12. I. N. Herstein, Noncommutative rings, Carus Mathematical Monographs, No. 15, Math. Assoc. Amer., 1968.

13. D. G. Johnson and J. Kist, Prime ideals in vector lattices, Canad. J. Math., 14 (1962), 517-528.

14. R. E. Johnson, Extended centralizer of a ring over a module, Proc. Amer. Math. Soc., 2 (1951), 891-895.

15. I. Kaplansky, Projective modules, Ann. Math., 68 (1958), 372-377.

16. - Algebraic and analytic aspects of operator algebras, Regional conference series in math., No. 1, Amer. Math. Soc., Providence, R.I., 1970.

17. J. Lambek, Lectures on Rings and Modules, Ginn (Blaisdell), Boston, 1966.

18. W. S. Martindale, 3rd, On semiprime P.I. rings, Proc. Amer. Math. Soc., 40 (1973), 365-369.

19. G. Renault, Anneaux reduits non commutatifs, J. Math. Pures Appl., 4 (1967), 203-214.

20. S. A. Steinberg, Quoiient rings of a class of lattice-ordered rings, Canad. J. Math., 25 (1973), 627-645. 
21. Y. Utumi, On quotient rings, Osaka Math. J., 8 (1956), 1-18.

22. - On rings of which any one-sided quotient rings are two-sided, Proc. Amer. Math. Soc., 14 (1963), 141-147.

23. R. Wiegand, Endomorphism rings of ideals in a commutative regular ring, Proc, Amer, Math. Soc., 23 (1969), 442-449.

24. E. T. Wong, Regular rings and integral extensions of a regular ring, Proc. Amer. Math. Soc., 33 (1972), 313-315.

25. E. T. Wong and R. E. Johnson, Self-injective rings, Canad. Math. Bull., 2 (1959), 167-173.

Received August 14, 1972 and in revised form February 1, 1973.

UNIVERSITY OF TOLEDO 


\section{PACIFIC JOURNAL OF MATHEMATICS}

\section{EDITORS}

RICHARD ARENS (Managing Editor)

University of California

Los Angeles, California 90024

\section{R. A. Beaumont}

University of Washington

Seattle, Washington 98105

\section{J. DuGundJI*}

Department of Mathematics

University of Southern California

Los Angeles, California 90007

D. Gilbarg and J. Milgram

Stanford University

Stanford, California 94305

ASSOCIATE EDITORS
E. F. BECKENBACH
B. H. NeumanN
F. WOLF
K. YoSHIDA

\section{SUPPORTING INSTITUTIONS}

UNIVERSITY OF BRITISH COLUMBIA

CALIFORNIA INSTITUTE OF TECHNOLOGY

UNIVERSITY OF CALIFORNIA

MONTANA STATE UNIVERSITY

UNIVERSITY OF NEVADA

NEW MEXICO STATE UNIVERSITY

OREGON STATE UNIVERSITY

UNIVERSITY OF OREGON

OSAKA UNIVERSITY

\author{
UNIVERSITY OF SOUTHERN CALIFORNIA \\ STANFORD UNIVERSITY \\ UNIVERSITY OF TOKYO \\ UNIVERSITY OF UTAH \\ WASHINGTON STATE UNIVERSITY \\ UNIVERSITY OF WASHINGTON \\ AMERICAN MATHEMATICAL SOCIETY \\ NAVAL WEAPONS CENTER
}

The Supporting Institutions listed above contribute to the cost of publication of this Journal, but they are not owners or publishers and have no responsibility for its content or policies.

Mathematical papers intended for publication in the Pacific Journal of Mathematics should be in typed form or offset-reproduced, (not dittoed), double spaced with large margins. Underline Greek letters in red, German in green, and script in blue. The first paragraph or two must be capable of being used separately as a synopsis of the entire paper. Items of the bibliography should not be cited there unless absolutely necessary, in which case they must be identified by author and Journal, rather than by item number. Manuscripts, in duplicate if possible, may be sent to any one of the four editors. Please classify according to the scheme of Math. Rev. Index to Vol. 39. All other communications to the editors should be addressed to the managing editor, or Elaine Barth, University of California, Los Angeles, California, 90024.

50 reprints are provided free for each article; additional copies may be obtained at cost in multiples of 50 .

The Pacific Journal of Mathematics is issued monthly as of January 1966. Regular subscription rate: $\$ 48.00$ a year (6 Vols., 12 issues). Special rate: $\$ 24.00$ a year to individual members of supporting institutions.

Subscriptions, orders for back numbers, and changes of address should be sent to Pacific Journal of Mathematics, 103 Highland Boulevard, Berkeley, California, 94708.

\section{PUBLISHED BY PACIFIC JOURNAL OF MATHEMATICS, A NON-PROFIT CORPORATION}

Printed at Kokusai Bunken Insatsusha (International Academic Printing Co., Ltd.), 270, 3-chome Totsuka-cho, Shinjuku-ku, Tokyo 160, Japan.

* C. R. DePrima California Institute of Technology, Pasadena, CA 91109, will replace J. Dugundji until August 1974.

Copyright (C) 1973 by

Pacific Journal of Mathematics

All Rights Reserved 


\section{Pacific Journal of Mathematics}

\section{Vol. 49, No. $2 \quad$ June, 1973}

Wm. R. Allaway, On finding the distribution function for an orthogonal polynomial

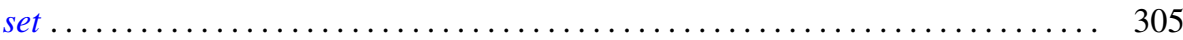

Eric Amar, Sur un théorème de Mooney relatif aux fonctions analytiques bornées... . 311

Robert Morgan Brooks, Analytic structure in the spectrum of a natural system . . . . 315

Bahattin Cengiz, On extremely regular function spaces . . . . . . . . . . . . . . 335

Kwang-nan Chow and Moses Glasner, Atoms on the Royden boundary . . . . . . . . . 339

Paul Frazier Duvall, Jr. and Jim Maxwell, Tame $Z^{2}$-actions on $E^{n} \ldots \ldots \ldots \ldots \ldots . .349$

Allen Roy Freedman, On the additivity theorem for $n$-dimensional asymptotic

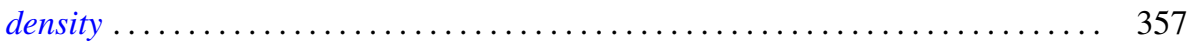

John Griffin and Kelly Denis McKennon, Multipliers and the group $L_{p}$-algebras . . . 365

Charles Lemuel Hagopian, Characterizations of $\lambda$ connected plane continua ....... 371

Jon Craig Helton, Bounds for products of interval functions . . . . . . . . . . . 377

Ikuko Kayashima, On relations between Nörlund and Riesz means . . . . . . . . . . 391

Everett Lee Lady, Slender rings and modules . . . . . . . . . . . . . . . . . . 397

Shozo Matsuura, On the Lu Qi-Keng conjecture and the Bergman representative

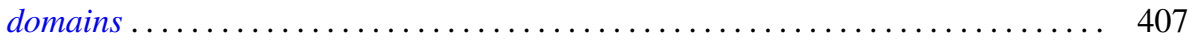

Stephen H. McCleary, The lattice-ordered group of automorphisms of an $\alpha$-set . . . 417

Stephen H. McCleary, o-2-transitive ordered permutation groups .......... 425

Stephen H. McCleary, o-primitive ordered permutation groups. II . . . . . . . . . 431

Richard Rochberg, Almost isometries of Banach spaces and moduli of planar

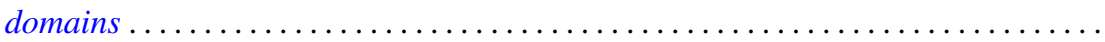

R. F. Rossa, Radical properties involving one-sided ideals . . . . . . . . . . . . . 467

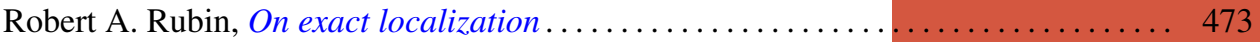

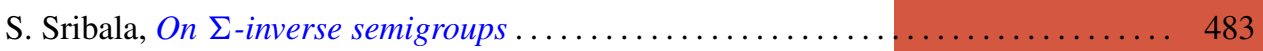

H. M. (Hari Mohan) Srivastava, On the Konhauser sets of biorthogonal polynomials suggested by the Laguerre polynomials ...................... 489

Stuart A. Steinberg, Rings of quotients of rings without nilpotent elements . ...... 493

Daniel Mullane Sunday, The self-equivalences of an $H$-space . . ............ 507

W. J. Thron and Richard Hawks Warren, On the lattice of proximities of $\check{C} e c h$

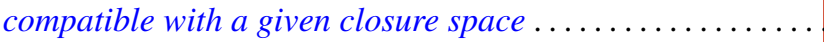

Frank Uhlig, The number of vectors jointly annihilated by two real quadratic forms determines the inertia of matrices in the associated pencil .

Frank Uhlig, On the maximal number of linearly independent real vectors annihilated simultaneously by two real quadratic forms ..............

Frank Uhlig, Definite and semidefinite matrices in a real symmetric matrix pencil . . 561

Arnold Lewis Villone, Self-adjoint extensions of symmetric differential operators . . . 569

Cary Webb, Tensor and direct products . ....................... 579

James Victor Whittaker, On normal subgroups of differentiable

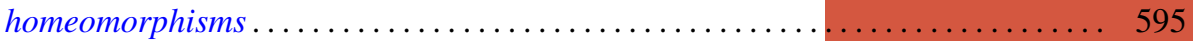

Jerome L. Paul, Addendum to: "Sequences of homeomorphisms which converge to

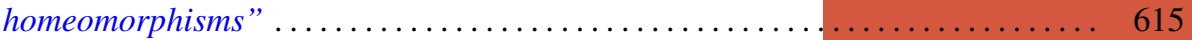

David E. Fields, Correction to: “Dimension theory in power series rings” ........ 616

Peter Michael Curran, Correction to: “Cohomology of finitely presented groups”. . . 617

Billy E. Rhoades, Correction to: “Commutants of some Hausdorff matrices” ...... 617

Charles W. Trigg, Corrections to: "Versum sequences in the binary system” ...... 619 